PROCEEDINGS OF THE

AMERICAN MATHEMATICAL SOCIETY

Volume 130, Number 10, Pages 2893-2904

S 0002-9939(02)06690-X

Article electronically published on May 1, 2002

\title{
CONSTRUCTION OF ORTHONORMAL WAVELETS USING KAMPÉ DE FÉRIET FUNCTIONS
}

\author{
AHMED I. ZAYED
}

(Communicated by David R. Larson)

\begin{abstract}
One of the main aims of this paper is to bridge the gap between two branches of mathematics, special functions and wavelets. This is done by showing how special functions can be used to construct orthonormal wavelet bases in a multiresolution analysis setting. The construction uses hypergeometric functions of one and two variables and a generalization of the latter, known as Kampé de Fériet functions. The mother wavelets constructed by this process are entire functions given by rapidly converging power series that allow easy and fast numerical evaluation. Explicit representation of wavelets facilitates, among other things, the study of the analytic properties of wavelets.
\end{abstract}

\section{INTRODUCTION}

Special functions of mathematical physics, such as Legendre, Hermite, Laguerre, and hypergeometric functions, have been around for more than two centuries and have been used successfully in many applications in applied mathematics, physics and engineering. On the other hand, wavelet analysis has emerged in the last decade not only as another powerful mathematical tool for applications, but also as a deep and profound mathematical theory. Up until now, these two branches of mathematics, special functions and wavelets, have not had much in common.

One of the main aims of this paper is to bridge this gap by showing how special functions can be used to construct orthonormal wavelet bases in a multiresolution analysis setting. The construction uses hypergeometric functions of one and two variables and a generalization of the latter, known as Kampé de Fériet functions. The mother wavelets constructed by this process are entire functions given by rapidly converging power series that allow easy and fast numerical evaluation. Moreover, explicit representation of wavelets facilitates, among other things, the study of the analytic properties of wavelets.

The paper is organized as follows. In Section 2, we introduce the notation and special functions that will be used in the sequel. For lack of space, we shall assume that the reader is familiar with wavelets and multiresolution analyses. The main result is presented in Section 3 as a theorem.

Received by the editors November 8, 2000.

2000 Mathematics Subject Classification. Primary 42C40, 33C20; Secondary 42C15, 33E20.

Key words and phrases. Orthonormal wavelets, bandlimited wavelets, multiresolution analysis, special functions, hypergeometric functions, Kampé de Fériet functions. 


\section{Preliminaries}

We adopt the following Pochhammer notation:

$$
(a)_{0}=1, \quad(a)_{n}=a(a+1) \ldots(a+n-1)=\frac{\Gamma(a+n)}{\Gamma(a)},
$$

where $n=1,2, \ldots$, and $a$ is a complex number. The generalized hypergeometric series ${ }_{p} F_{q}$ is defined as

$$
\begin{aligned}
{ }_{p} F_{q}\left[\begin{array}{ccc}
a_{1}, & \ldots & a_{p} ; \\
b_{1}, & \ldots & b_{q}
\end{array}\right] & ={ }_{p} F_{q}\left(a_{1}, \ldots, a_{p} ; b_{1}, \ldots, b_{q} ; z\right) \\
& =\sum_{n=0}^{\infty} \frac{\left(a_{1}\right)_{n}\left(a_{2}\right)_{n} \ldots\left(a_{p}\right)_{n}}{\left(b_{1}\right)_{n}\left(b_{2}\right)_{n} \ldots\left(b_{q}\right)_{n}} \frac{z^{n}}{n !}, \quad z \text { is complex. }
\end{aligned}
$$

The constants $a_{i}, i=1, \ldots, p$, and $b_{j}, j=1, \ldots, q$, are complex numbers and none of the $b_{j}$ 's is a negative integer. The series converges for all finite $z$ if $p \leq$ $q$, converges for $|z|<1$ if $p=q+1$, converges for $|z| \leq 1$ if $p=q+1$ and $\operatorname{Re}\left\{\sum_{i=1}^{q} b_{i}-\sum_{i=1}^{p} a_{i}\right\}>0$, and diverges for all $z \neq 0$ if $p>q+1$.

Hypergeometric series in two variables can be defined similarly. We shall introduce the following generalization of the hypergeometric function of two variables, which was originally introduced by Kampé de Fériet (see ([3, Ch. 1]) and slightly generalized by Srivastava and Panda (see [6, p. 63]).

The generalized Kampé de Fériet function of two variables is defined as

$$
F_{l: m ; n}^{p: q ; k}\left[\begin{array}{cc}
\left(a_{p}\right):\left(b_{q}\right) ; & \left(c_{k}\right) ; \\
\left(\alpha_{l}\right):\left(\beta_{m}\right) ; & \left(\gamma_{n}\right) ;
\end{array}\right]=\sum_{r, s=0}^{\infty} \frac{\prod_{j=1}^{p}\left(a_{j}\right)_{r+s} \prod_{j=1}^{q}\left(b_{j}\right)_{r} \prod_{j=1}^{k}\left(c_{j}\right)_{s}}{\prod_{j=1}^{l}\left(\alpha_{j}\right)_{r+s} \prod_{j=1}^{m}\left(\beta_{j}\right)_{r} \prod_{j=1}^{n}\left(\gamma_{j}\right)_{s}} \frac{x^{r}}{r !} \frac{y^{s}}{s !},
$$

where $\prod_{j=1}^{N} z_{j}=z_{1} z_{2} \ldots z_{N}$, and none of the parameters in the denominator is a negative integer. The series converges for all $x$ and $y$ if $p+q<l+m+1$ and $p+k<$ $l+n+1$. If $p+q=l+m+1$ and $p+k<l+n+1$, then the series converges for $|x| \leq 1$ and all $y$, provided that

$$
\operatorname{Re}\left\{\sum_{j=1}^{l} \alpha_{j}+\sum_{j=1}^{m} \beta_{j}-\sum_{j=1}^{p} a_{j}-\sum_{j=1}^{q} b_{j}\right\}>0
$$

If $p+q<l+m+1$ and $p+k=l+n+1$, then the series converges for $|y| \leq 1$ and all $x$, provided that the same condition holds but with the $\beta$ 's replaced by $\gamma$ 's and the $b$ 's replaced by $c$ 's.

Young's function [7] of order $\nu(\nu \geq 0)$, which will be denoted by $\mathbf{Y}_{\nu}$, is defined by

$$
\mathbf{Y}_{\nu}(z)=z^{\nu} \sum_{k=0}^{\infty} \frac{(-1)^{k} z^{2 k}}{\Gamma(\nu+2 k+1)}=\frac{z^{\nu}}{\Gamma(\nu+1)}{ }_{1} F_{2}\left(1 ; \frac{\nu+1}{2}, \frac{\nu+2}{2} ;-\frac{z^{2}}{4}\right)
$$

This function should be distinguished from the Bessel function of the second kind and order $\nu$, which is usually denoted by $Y_{\nu}$. Clearly, $\mathbf{Y}_{0}(z)=\cos z$ and $\mathbf{Y}_{1}(z)=$ $\sin z$. 
Another important and related special function that will be used in the sequel is the following integral of Young's function, $I_{\nu, \alpha}$, defined by

$$
\begin{aligned}
I_{\nu, \alpha}(x) & =\int x^{\alpha} \mathbf{Y}_{\nu}(x) d x=\sum_{k=0}^{\infty} \frac{(-1)^{k} x^{2 k+\nu+\alpha+1}}{(2 k+\nu+\alpha+1) \Gamma(\nu+2 k+1)} \\
& =\frac{x^{\nu+\alpha+1}}{(\nu+\alpha+1) \Gamma(\nu+1)}{ }_{2} F_{3}\left(1, \frac{\nu+\alpha+1}{2} ; \frac{\nu+1}{2}, \frac{\nu+2}{2}, \frac{\nu+\alpha+3}{2} ;-\frac{x^{2}}{4}\right) .
\end{aligned}
$$

In particular,

$$
\int \sqrt{x} \cos x d x=I_{0,1 / 2}(x) \quad \text { and } \quad \int \sqrt{x} \sin x d x=I_{1,1 / 2}(x) .
$$

Here we assume that the reader is familiar with the theory of wavelets and multiresolution analysis; see [1] for details. It is well known that a function $\phi(t)$ is an orthogonal scaling function of a multiresolution analysis if it satisfies the following conditions:

i) $\quad \sum_{k}|\widehat{\phi}(\omega+2 \pi k)|^{2}=1$,

ii) $\widehat{\phi}(\omega)=\left(\frac{1}{2} \sum_{k} c_{k} e^{i k \omega / 2}\right) \widehat{\phi}\left(\frac{\omega}{2}\right)=m\left(\frac{\omega}{2}\right) \widehat{\phi}\left(\frac{\omega}{2}\right)$, where $m\left(\frac{\omega}{2}\right)=\frac{1}{2} \sum_{k} c_{k} e^{i k \omega / 2}$.

iii) $\widehat{\phi}(\omega)$ is continuous at $\omega=0$ and $\widehat{\phi}(0)=1$.

The mother wavelet $\psi$ can be obtained from the relation

$$
\widehat{\psi}(\omega)=e^{i \omega / 2} \bar{m}\left(\frac{\omega}{2}+\pi\right) \widehat{\phi}\left(\frac{\omega}{2}\right) .
$$

\section{The MAIN RESUlt}

In this section we present the main result. But first we need the following lemmas. The first one, whose proof can be found in [8, 9, will play an important role in constructing the mother wavelets.

Lemma 1. Let $h$ be a function satisfying the following conditions:

1) $h \in L^{1}(R)$,

2) $0 \leq h$,

3) $\int_{-\infty}^{\infty} h(x) d x=1$,

4) $h(x)$ is even,

5) support $h \subset\left[-\frac{\pi}{3}, \frac{\pi}{3}\right]$.

Let

$$
g(w)=\int_{w-\pi}^{w+\pi} h(x) d x .
$$

Then $g(w)$ is a nonnegative, even, continuous function, with support in $\left[-\frac{4 \pi}{3}, \frac{4 \pi}{3}\right]$ and $g(w)=1$ on $\left[-\frac{2 \pi}{3}, \frac{2 \pi}{3}\right]$. Moreover, $\sum_{k=-\infty}^{\infty} g(w+2 \pi k)=1$. The function $\hat{\phi}(w)=\sqrt{g(w)}$ is an orthogonal scaling function of a multiresolution analysis. 
The scaling function constructed in the above lemma is clearly bandlimited; hence, so is its associated mother wavelet. This method of constructing bandlimited wavelets is related, yet different from the one developed by Hernandez and Weiss [5, Ch. 3].

\section{Lemma 2. Let}

$$
I=I(u, z)=\int_{0}^{u} \sqrt{1-x^{2}} \cos (z x) d x
$$

and

$$
J=J(u, z)=\int_{0}^{u} \sqrt{1-x^{2}} \sin (z x) d x
$$

where $0 \leq u \leq 1 / \sqrt{2}$ and $z$ is real. Then

$$
I=u \sqrt{1-u^{2}} F_{1: 0 ; 0}^{0: 2 ; 0}\left[\begin{array}{ccc}
-(-1 / 2),(1) ; \ldots & \ldots \\
(3 / 2): \ldots & \ldots & \ldots, B
\end{array}\right]
$$

and

$$
J=\left(\frac{z u^{2}}{2}\right) \sqrt{1-u^{2}} F_{1: 0 ; 1}^{0: 2 ; 1}\left[\begin{array}{ccc}
0:(-1 / 2),(1) ; & 1 ; \\
2: \ldots & (3 / 2) ; & A, B
\end{array}\right]
$$

where $A=u^{2} /\left(u^{2}-1\right)$ and $B=-u^{2} z^{2} / 4$.

In particular, if $u=1 / \sqrt{2}$

$$
I=\frac{1}{2} F_{1: 0 ; 0}^{0: 2 ; 0}\left[\begin{array}{ccc} 
& :(-1 / 2),(1) ; \ldots \ldots & \\
(3 / 2): \_ & \ldots & \ldots
\end{array}\right]
$$

and

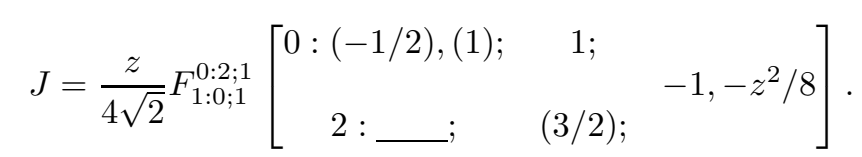

Proof. Expanding $\cos (z x)$ in a power series leads to

$$
I=\sum_{n=0}^{\infty} \frac{(-1)^{n} z^{2 n}}{(2 n) !} \int_{0}^{u} \sqrt{1-x^{2}} x^{2 n} d x
$$

Interchanging the summation and integration signs is permissible since the series converges uniformly for all $x$ and $z$ real. Setting $x^{2}=v$, we obtain

$$
I=\frac{1}{2} \sum_{n=0}^{\infty} \frac{(-1)^{n} z^{2 n}}{(2 n) !} \int_{0}^{u^{2}} \sqrt{1-v} v^{n-1 / 2} d v
$$

of formula 3.194-1 in [4, p. 284], which states that

$$
\int_{0}^{u} \frac{x^{\mu-1}}{(1+\beta x)^{\nu}} d x=\frac{u^{\mu}}{\mu}{ }_{2} F_{1}(\nu, \mu ; 1+\mu ;-\beta u), \quad \text { Re } \mu>0, \quad \arg (1+\beta u)<\pi,
$$


we have

$$
I=\sum_{n=0}^{\infty} \frac{(-1)^{n} z^{2 n} u^{2 n+1}}{(2 n+1) !}{ }_{2} F_{1}\left(-\frac{1}{2}, n+\frac{1}{2} ; n+\frac{3}{2} ; u^{2}\right)
$$

Using the transformation [2, p. 64]

$$
{ }_{2} F_{1}(a, b ; c ; w)=(1-w)^{-a} F_{1}(a, c-b ; c ; w /(w-1)),
$$

we get

$$
I=\left(1-u^{2}\right)^{1 / 2} \sum_{n=0}^{\infty} \frac{(-1)^{n} z^{2 n} u^{2 n+1}}{(2 n+1) !}{ }_{2} F_{1}\left(-1 / 2,1 ; n+3 / 2 ; u^{2} /\left(u^{2}-1\right)\right) .
$$

Expanding the hypergeometric function in a power series, we have

$$
I=\left(1-u^{2}\right)^{1 / 2} \sum_{n=0}^{\infty} \frac{(-1)^{n} z^{2 n} u^{2 n+1}}{(2 n+1) !} \sum_{k=0}^{\infty} \frac{(-1 / 2)_{k}(1)_{k}}{\left(n+\frac{3}{2}\right)_{k} k !} A^{k},
$$

where $A=u^{2} /\left(u^{2}-1\right)$. The expansion is valid for $|A|<1$. In fact, since the hypergeometric series of ${ }_{2} F_{1}(a, b ; c ; w)$ converges absolutely and uniformly for $|w| \leq$ 1 , provided that $\operatorname{Re}(c-a-b)>0$, it follows that for $0 \leq u \leq 1 / \sqrt{2}$, we can interchange the summation in (10) to obtain

$$
I=\left(1-u^{2}\right)^{1 / 2} \sum_{n, k=0}^{\infty} \frac{(-1)^{n} z^{2 n} u^{2 n+1}\left(-\frac{1}{2}\right)_{k}(1)_{k}}{(2 n+1) !\left(n+\frac{3}{2}\right)_{k} k !} A^{k} .
$$

By using the Legendre duplication formula

$$
(2 n+1) !=\Gamma(2 n+2)=\frac{2^{2 n+1}}{\sqrt{\pi}} \Gamma(n+1) \Gamma\left(n+\frac{3}{2}\right)=2^{2 n} n !(3 / 2)_{n},
$$

and noting that

$$
(n+3 / 2)_{k}=\frac{\Gamma(n+k+3 / 2)}{\Gamma(n+3 / 2)}=\frac{(3 / 2)_{n+k} \Gamma(3 / 2)}{\Gamma(n+3 / 2)}=\frac{(3 / 2)_{n+k}}{(3 / 2)_{n}},
$$

we have

$$
I=u\left(1-u^{2}\right)^{1 / 2} \sum_{n, k=0}^{\infty} \frac{\left(-\frac{1}{2}\right)_{k}(1)_{k}}{(3 / 2)_{n+k}} \frac{B^{n}}{n !} \frac{A^{k}}{k !}
$$

where $B=-z^{2} u^{2} / 2^{2}$. Thus, using the notation of Section 2 , we have

$$
I=u\left(1-u^{2}\right)^{1 / 2} F_{1: 0 ; 0}^{0: 2 ; 0}\left[\begin{array}{ccc}
:(-1 / 2),(1) ; \ldots & \ldots & \\
(3 / 2): \ldots & \ldots & \ldots
\end{array}\right] .
$$

The right-hand side is well defined even for $|A|=1$; see Section 2 .

As for $J$, we expand $\sin (z x)$ in a power series to obtain

$J=\sum_{n=0}^{\infty} \frac{(-1)^{n} z^{2 n+1}}{(2 n+1) !} \int_{0}^{u} \sqrt{1-x^{2}} x^{2 n+1} d x=\frac{1}{2} \sum_{n=0}^{\infty} \frac{(-1)^{n} z^{2 n+1}}{(2 n+1) !} \int_{0}^{u^{2}} \sqrt{1-v} v^{n} d v$ 
which, with the aid of (9), yields

$$
\begin{aligned}
J & =\frac{1}{2} \sum_{n=0}^{\infty} \frac{(-1)^{n} z^{2 n+1} u^{2 n+2}}{(2 n+1) !(n+1)}{ }_{2} F_{1}\left(-\frac{1}{2}, n+1 ; n+2 ; u^{2}\right) \\
& =\sum_{n=0}^{\infty} \frac{(-1)^{n} z^{2 n+1} u^{2 n+2}}{(2 n+2) !}{ }_{2} F_{1}\left(-\frac{1}{2}, n+1 ; n+2 ; u^{2}\right) .
\end{aligned}
$$

Expanding the hypergeometric function in a power series, we have

$$
\begin{aligned}
J & =\left(1-u^{2}\right)^{1 / 2} \sum_{n=0}^{\infty} \frac{(-1)^{n} z^{2 n+1} u^{2 n+2}}{(2 n+2) !}{ }_{2} F_{1}\left(-1 / 2,1 ; n+2 ; \frac{u^{2}}{u^{2}-1}\right) \\
& =\left(1-u^{2}\right)^{1 / 2} \sum_{k, n=0}^{\infty} \frac{(-1)^{n} z^{2 n+1} u^{2 n+2}}{(2 n+2) !} \frac{(-1 / 2)_{k}(1)_{k}}{(n+2)_{k} k !} A^{k} .
\end{aligned}
$$

But

$$
\begin{aligned}
(2 n+2) ! & =\Gamma(2 n+3)=\frac{1}{\sqrt{\pi}} 2^{2 n+2} \Gamma(n+2) \Gamma(n+3 / 2) \\
& =2^{2 n+1} \Gamma(n+2)(3 / 2)_{n}
\end{aligned}
$$

and

$$
(n+2)_{k}=\frac{\Gamma(n+k+2)}{\Gamma(n+2)}=\frac{(2)_{n+k}}{\Gamma(2) \Gamma(n+2)}
$$

hence

$$
\begin{aligned}
J & =\left(1-u^{2}\right)^{1 / 2} \sum_{k, n=0}^{\infty} \frac{(-1)^{n} z^{2 n+1} u^{2 n+2}(-1 / 2)_{k}(1)_{k}}{2^{2 n+1}(2)_{n+k}(3 / 2)_{n}} \frac{A^{k}}{k !} \\
& =\left(\frac{z u^{2}}{2}\right)\left(1-u^{2}\right)^{1 / 2} \sum_{k, n=0}^{\infty} \frac{(-1 / 2)_{k}(1)_{k}(1)_{n}}{(2)_{n+k}(3 / 2)_{n}} \frac{A^{k}}{k !} \frac{B^{n}}{n !}
\end{aligned}
$$

where $B=-u^{2} z^{2} / 4$. Therefore

$$
J=\left(\frac{z u^{2}}{2}\right)\left(1-u^{2}\right)^{1 / 2} F_{1: 0 ; 1}^{0: 2 ; 1}\left[\begin{array}{ccc}
0:(-1 / 2),(1) ; & 1 ; \\
2: \ldots \ldots & (3 / 2) ;
\end{array}\right]
$$

Now we state and prove our main theorem.

Theorem 1. The functions $\phi_{1}(t)$ and $\phi_{2}(t)$ given by

$$
\phi_{1}(t)=\frac{\sin \frac{2 \pi t}{3}}{\pi t}+\frac{\sqrt{3}}{\pi(2 t)^{3 / 2}}\left[\mathbf{Y}_{3 / 2}\left(\frac{2 \pi t}{3}\right) \cos \left(\frac{2 \pi t}{3}\right)-\mathbf{Y}_{5 / 2}\left(\frac{2 \pi t}{3}\right) \sin \left(\frac{2 \pi t}{3}\right)\right]
$$


and

$$
\begin{aligned}
\phi_{2}(t)= & \frac{3}{\sqrt{2}}\left\{\frac{\cos (4 \pi t / 3)}{\pi^{2} t^{2}}+\frac{\sin \pi t}{3 \pi t}-\frac{\cos \pi t}{\pi^{2} t^{2}}\right\} \\
& +\frac{1}{3 \sqrt{2}} \cos \frac{2 \pi t}{3} F_{1: 0 ; 0}^{0: 2 ; 0}\left[-:(-1 / 2),(1) ;-1,-1,-\pi^{2} t^{2} / 36\right] \\
& +\frac{\sin (2 \pi t / 3)}{\pi t}\left\{1-\frac{\pi^{2} t^{2}}{18 \sqrt{2}} F_{1: 0 ; 1}^{0: 2 ; 1}\left[\begin{array}{cc}
0:(-1 / 2),(1) ; & 1 ; \\
2: \ldots & (3 / 2) ;
\end{array}\right]\right\}
\end{aligned}
$$

are orthogonal scaling functions of a multiresolution analysis and their associated mother wavelets are given respectively by

$$
\begin{aligned}
\psi_{1}\left(t+\frac{1}{2}\right)=\frac{\sqrt{3 / 2}}{(\pi t)^{3 / 2}}\left\{\left(\cos \left(\frac{2 \pi t}{3}\right) I_{0,1 / 2}\left(\frac{2 \pi t}{3}\right)-\sin \left(\frac{2 \pi t}{3}\right) I_{1,1 / 2}\left(\frac{2 \pi t}{3}\right)\right)\right. \\
\text { (12) } \left.\quad+\sqrt{\frac{\pi}{8}}\left(\cos \left(\frac{4 \pi t}{3}\right) \mathbf{Y}_{3 / 2}\left(\frac{4 \pi t}{3}\right)-\sin \left(\frac{4 \pi t}{3}\right) \mathbf{Y}_{5 / 2}\left(\frac{4 \pi t}{3}\right)\right)\right\}
\end{aligned}
$$

and

$$
\begin{aligned}
\psi_{2}\left(t+\frac{1}{2}\right)= & \frac{3}{\sqrt{2}}\left\{\frac{\sin \pi t}{3 \pi t}+\frac{\cos \pi t-\cos (2 \pi t / 3)}{\pi^{2} t^{2}}+\frac{\cos (8 \pi t / 3)-\cos 2 \pi t}{2 \pi^{2} t^{2}}+\frac{\sin 2 \pi t}{3 \pi t}\right\} \\
& +\frac{1}{3 \sqrt{2}} \cos \left(\frac{4 \pi t}{3}\right)[\Phi(\tau)+2 \Phi(2 \tau)] \\
& +\frac{\pi t}{18 \sqrt{2}} \sin \left(\frac{4 \pi t}{3}\right)[\Psi(\tau)-4 \Psi(2 \tau)],
\end{aligned}
$$

where $\tau=\frac{\sqrt{2} \pi t}{3}$ and

$$
\Phi(\tau)=F_{1: 0 ; 0}^{0: 2 ; 0}\left[\begin{array}{l}
-:(-1 / 2),(1) ; \quad \ldots ; \\
(3 / 2): \_; \ldots ;
\end{array} \quad-1,-\frac{\tau^{2}}{8}\right]
$$

and

$$
\Psi(\tau)=F_{1: 0 ; 1}^{0: 2 ; 1}\left[\begin{array}{ccc}
0:(-1 / 2),(1) ; & 1 ; & \\
2: \ldots \ldots & (3 / 2) ; & -1,-\frac{\tau^{2}}{8}
\end{array}\right] .
$$

Proof. We start with $\phi_{1}$. In Lemma 1, let $h(t)=\frac{3}{2 \pi} \chi_{\left[-\frac{\pi}{3}, \frac{\pi}{3}\right]}(t)$ be the characteristic function of the interval $\left[-\frac{\pi}{3}, \frac{\pi}{3}\right]$ and define

$$
g(w)=\int_{w-\pi}^{w+\pi} h(t) d t .
$$

With straightforward calculations, one can show that

$$
g(w)= \begin{cases}0, & \text { if }|w| \geq \frac{4 \pi}{3}, \\ \frac{3}{2 \pi} w+2 & \text { if }-\frac{4 \pi}{3} \leq w \leq-\frac{2 \pi}{3}, \\ 1 & \text { if }|w| \leq \frac{2 \pi}{3} \\ -\frac{3}{2 \pi} w+2 & \text { if } \frac{2 \pi}{3} \leq w \leq \frac{4 \pi}{3} .\end{cases}
$$


Since $g(w)$ is nonnegative, by Lemma 1 we define $\hat{\phi}_{1}(w)=\sqrt{g(w)}$; hence

$$
\begin{aligned}
\sum_{k=-\infty}^{\infty}\left|\hat{\phi}_{1}(w+2 \pi k)\right|^{2} & =\sum_{k=-\infty}^{\infty} g(w+2 \pi k) \\
& =\sum_{k=-\infty}^{\infty} \int_{w+2 \pi k-\pi}^{w+2 \pi k+\pi} h(t) d t=\int_{-\infty}^{\infty} h(t) d t=1 .
\end{aligned}
$$

Thus, $\left\{\phi_{1}(t-n)\right\}$ is orthonormal in $L^{2}(\mathbb{R})$. If we define $m\left(\frac{w}{2}\right)$ as the $4 \pi$-periodic extension of $\hat{\phi}_{1}(w)$, it will follow that $\hat{\phi}_{1}$ satisfies the dilation equation (4)ii), as well as (4)ii). Therefore, $\phi_{1}(t)$ is an orthogonal scaling function of a multiresolution analysis. To obtain $\phi_{1}$ in closed form, we use the inversion formula for the Fourier transform, which in view of the fact that $\hat{\phi}_{1}$ is even, yields

$$
\begin{aligned}
\phi_{1}(t) & =\frac{1}{2 \pi} \int_{-\infty}^{\infty} \hat{\phi}_{1}(w) e^{-i t w} d w=\frac{1}{\pi} \int_{0}^{\frac{4 \pi}{3}} \hat{\phi}_{1}(w) \cos (w t) d w \\
& =\frac{1}{\pi}\left\{\int_{0}^{\frac{2 \pi}{3}} \cos (w t) d w+\int_{\frac{2 \pi}{3}}^{\frac{4 \pi}{3}} \sqrt{2-\frac{3 w}{2 \pi}} \cos (w t) d w\right\} \\
& =\frac{\sin \left(\frac{2 t \pi}{3}\right)}{\pi t}+\frac{2}{3} \int_{1}^{2} \sqrt{2-\gamma} \cos \left(\frac{2 \pi t \gamma}{3}\right) d \gamma
\end{aligned}
$$

By setting $w=\gamma-1$ and $c=\frac{2 \pi t}{3}$, we obtain

$$
\begin{aligned}
\phi_{1}(t) & =\frac{\sin \left(\frac{2 \pi t}{3}\right)}{\pi t}+\frac{2}{3} \int_{0}^{1} \sqrt{1-w} \cos [c(w+1)] d w \\
& =\frac{\sin \left(\frac{2 \pi t}{3}\right)}{\pi t}+\frac{2}{3}\left[\cos c \int_{0}^{1} \sqrt{1-w} \cos (c w) d w-\sin c \int_{0}^{1} \sqrt{1-w} \sin (c w) d w\right] .
\end{aligned}
$$

But from 9

$$
\begin{aligned}
& \int_{0}^{1} \sqrt{1-w} \cos (c w) d w=\frac{\sqrt{\pi}}{2} \frac{\mathbf{Y}_{3 / 2}(c)}{c^{3 / 2}} \text { and } \\
& \int_{0}^{1} \sqrt{1-w} \sin (c w) d w=\frac{\sqrt{\pi}}{2} \frac{\mathbf{Y}_{5 / 2}(c)}{c^{3 / 2}}
\end{aligned}
$$

and the substitution of (16) into (15) yields (11).

To derive $\psi_{1}(t)$ explicitly, we appeal to Eq. (5). Because of the symmetry of $e^{-i w / 2} \hat{\psi}_{1}(w)$, it suffices to consider its restriction to the positive real axis that is given by

$$
e^{-i w / 2} \hat{\psi}_{1}(w)= \begin{cases}0, & 0 \leq w \leq 2 \pi / 3 \\ \sqrt{\frac{3 w}{2 \pi}-1}, & 2 \pi / 3 \leq w \leq 4 \pi / 3 \\ \sqrt{-\frac{3 w}{4 \pi}+2}, & 4 \pi / 3 \leq w \leq 8 \pi / 3 \\ 0, & 8 \pi / 3 \leq w\end{cases}
$$


By taking the inverse Fourier transform of $e^{-i w / 2} \hat{\psi}_{1}(w)$, we obtain

$$
\begin{aligned}
& \psi_{1}\left(t+\frac{1}{2}\right)=\frac{1}{\pi} \int_{0}^{8 \pi / 3} e^{-i w / 2} \hat{\psi}_{1}(w) \cos (t w) d w \\
& =\frac{1}{\pi}\left\{\int_{2 \pi / 3}^{4 \pi / 3} \sqrt{\frac{3 w}{2 \pi}-1} \cos (t w) d w+\int_{4 \pi / 3}^{8 \pi / 3} \sqrt{-\frac{3 w}{4 \pi}+2} \cos (t w) d w\right\} \\
& =\frac{2}{3} \int_{1}^{2} \sqrt{\gamma-1} \cos \left(\frac{2 \pi \gamma t}{3}\right) d \gamma+\frac{4}{3} \int_{1}^{2} \sqrt{2-\gamma} \cos \left(\frac{4 \pi \gamma t}{3}\right) d \gamma \\
& =\frac{2}{3} \int_{0}^{1} \sqrt{u} \cos (\alpha u+\alpha) d u+\frac{4}{3} \int_{0}^{1} \sqrt{1-u} \cos (\beta u+\beta) d u
\end{aligned}
$$

where $\alpha=2 \pi t / 3$ and $\beta=2 \alpha$. Thus, in view of Eq. (3), we have

$$
\begin{aligned}
\psi_{1}\left(t+\frac{1}{2}\right)=\frac{2}{3}\{ & \cos \alpha \int_{0}^{1} \sqrt{u} \cos (\alpha u) d u-\sin \alpha \int_{0}^{1} \sqrt{u} \sin (\alpha u) d u \\
& \left.+2 \cos \beta \int_{0}^{1} \sqrt{1-u} \cos (\beta u) d u-2 \sin \beta \int_{0}^{1} \sqrt{1-u} \sin (\beta u) d u\right\} \\
=\frac{2}{3}\{ & \frac{1}{\alpha^{3 / 2}}\left(\cos \alpha I_{0,1 / 2}(\alpha)-\sin \alpha I_{1,1 / 2}(\alpha)\right) \\
& \left.+\frac{\pi}{\beta^{3 / 2}}\left(\cos \beta \mathbf{Y}_{3 / 2}(\beta)-\sin \beta \mathbf{Y}_{5 / 2}(\beta)\right)\right\}
\end{aligned}
$$

which is (12).

As for $\phi_{2}$, we take

$$
h(t)= \begin{cases}\frac{9}{\pi^{2}} x+\frac{3}{\pi} & \text { if } \quad-\frac{\pi}{3} \leq x \leq 0 \\ -\frac{9}{\pi^{2}} x+\frac{3}{\pi} & \text { if } \quad 0 \leq x \leq \frac{\pi}{3} .\end{cases}
$$

Then it is easy to verify that $h$ satisfies all the conditions of Lemma 1 . Set

$$
g(w)=\int_{w-\pi}^{w+\pi} h(t) d t
$$

With some easy calculations, we have

$$
g(w) \begin{cases}0, & w \leq-\frac{4 \pi}{3} \\ \frac{9}{2 \pi^{2}} w^{2}+\frac{12 w}{\pi}+8, & -\frac{4 \pi}{3} \leq w \leq-\pi \\ -\frac{9}{2 \pi^{2}} w^{2}-\frac{6}{\pi} w-1, & -\pi \leq w \leq-\frac{2 \pi}{3} \\ 1, & -\frac{2 \pi}{3} \leq w \leq \frac{2 \pi}{3} \\ -\frac{9}{2 \pi^{2}} w^{2}+\frac{6 w}{\pi}-1, & \frac{2 \pi}{3} \leq w \leq \pi \\ \frac{9}{2 \pi^{2}} w^{2}-\frac{12 w}{\pi}+8, & \pi \leq w \leq \frac{4 \pi}{3} \\ 0, & \frac{4 \pi}{3} \leq w\end{cases}
$$

It follows as before that $\phi_{2}(t)$ is an orthogonal scaling function of a multiresolution analysis. To obtain $\phi_{2}(t)$ explicitly, we have

$$
\begin{aligned}
\phi_{2}(t) & =\frac{1}{2 \pi} \int_{-\infty}^{\infty} \hat{\phi}_{2}(w) e^{-i t w} d w=\frac{1}{\pi} \int_{0}^{\frac{4 \pi}{3}} \hat{\phi}_{2}(w) \cos (t w) d w \\
& =\frac{1}{\pi} \int_{0}^{\frac{2 \pi}{3}} \cos (t w) d w+I_{2}+I_{3}=\frac{\sin (2 \pi t / 3)}{\pi t}+I_{2}+I_{3},
\end{aligned}
$$


where

$$
I_{2}=\frac{1}{\pi} \int_{\frac{2 \pi}{3}}^{\pi} \sqrt{-\frac{9}{2 \pi^{2}} w^{2}+\frac{6 w}{\pi}-1} \cos (t w) d w
$$

and

$$
I_{3}=\frac{1}{\pi} \int_{\pi}^{\frac{4 \pi}{3}} \sqrt{\frac{9}{2 \pi^{2}} w^{2}-\frac{12 w}{\pi}+8} \cos (t w) d w
$$

To evaluate $I_{3}$, we have

$$
\begin{aligned}
I_{3} & =\frac{3}{\sqrt{2} \pi^{2}} \int_{\pi}^{\frac{4 \pi}{3}} \sqrt{w^{2}-\frac{8 \pi}{3} w+\frac{16 \pi^{2}}{9}} \cos (t w) d w \\
& =\frac{3}{\sqrt{2} \pi^{2}} \int_{\pi}^{\frac{4 \pi}{3}} \sqrt{\left(w-\frac{4 \pi}{3}\right)^{2}} \cos (t w) d w=\frac{3}{\sqrt{2} \pi^{2}} \int_{\pi}^{\frac{4 \pi}{3}}\left(w-\frac{4 \pi}{3}\right) \cos (t w) d w \\
& =\frac{3}{\sqrt{2}}\left[\frac{\cos (4 \pi t / 3)}{\pi^{2} t^{2}}+\frac{\sin \pi t}{3 \pi t}-\frac{\cos \pi t}{\pi^{2} t^{2}}\right] .
\end{aligned}
$$

Evaluating $I_{2}$ is more difficult. To this end, we have

$$
\begin{aligned}
I_{2} & =\frac{3}{\sqrt{2} \pi^{2}} \int_{\frac{2 \pi}{3}}^{\pi} \sqrt{\frac{2 \pi^{2}}{9}-\left(w-\frac{2 \pi}{3}\right)^{2}} \cos (t w) d w \\
& =\frac{3}{\sqrt{2} \pi^{2}} \int_{0}^{\frac{\pi}{3}} \sqrt{\frac{2 \pi^{2}}{9}-\gamma^{2}} \cos t\left(\gamma+\frac{2 \pi}{3}\right) d \gamma \\
& =\frac{\sqrt{2}}{3} \int_{0}^{\frac{1}{\sqrt{2}}} \sqrt{1-x^{2}} \cos \left(\tau x+\frac{2 \pi t}{3}\right) d x=\frac{\sqrt{2}}{3}\left[\cos \frac{2 \pi t}{3} I_{2}^{\prime}-\sin \frac{2 \pi t}{3} I_{2}^{\prime \prime}\right],
\end{aligned}
$$

where $\tau=\frac{\sqrt{2} \pi t}{3}$ and

$$
I_{2}^{\prime}(\tau)=\int_{0}^{\frac{1}{\sqrt{2}}} \sqrt{1-x^{2}} \cos (\tau x) d x \quad \text { and } \quad I_{2}^{\prime \prime}(\tau)=\int_{0}^{\frac{1}{\sqrt{2}}} \sqrt{1-x^{2}} \sin (\tau x) d x
$$

Now by (7) and (8) we obtain

$$
\begin{aligned}
& I_{2}=\frac{1}{3 \sqrt{2}} \cos \left(\frac{2 \pi t}{3}\right) F_{1: 0 ; 0}^{0: 2 ; 0}\left[\begin{array}{ccc}
:(-1 / 2),(1) ; \quad \ldots ; & -1,-\pi^{2} t^{2} / 36 \\
(3 / 2): \ldots \ldots & \ldots
\end{array}\right] \\
& -\frac{\pi t}{18 \sqrt{2}} \sin \left(\frac{2 \pi t}{3}\right) F_{1: 0 ; 1}^{0: 2 ; 1}\left[\begin{array}{ccc}
0:(-1 / 2),(1) ; & 1 ; & -1,-\pi^{2} t^{2} / 36 \\
2: \ldots \ldots & (3 / 2) ;
\end{array}\right] \text {. }
\end{aligned}
$$


Now we find the associated mother wavelet $\psi_{2}$ :

$$
\begin{aligned}
\psi_{2}\left(t+\frac{1}{2}\right)= & \frac{1}{2 \pi} \int_{-\infty}^{\infty} e^{-i w / 2} \hat{\psi}_{2}(w) e^{-i t w} d w=\frac{1}{\pi} \int_{0}^{\frac{8 \pi}{3}} e^{-i w / 2} \hat{\psi}_{2}(w) \cos (t w) d w \\
= & \frac{1}{\pi}\left\{\int_{\frac{2 \pi}{3}}^{\pi} \sqrt{\frac{9 w^{2}}{2 \pi^{2}}-\frac{6 w}{\pi}+2} \cos (t w) d w\right. \\
& +\int_{\pi}^{\frac{4 \pi}{3}} \sqrt{-\frac{9}{2 \pi^{2}} w^{2}+\frac{12 w}{\pi}-7} \cos (t w) d w \\
& +\int_{\frac{4 \pi}{3}}^{2 \pi} \sqrt{-\frac{9 w^{2}}{8 \pi^{2}}+\frac{3 w}{\pi}-1} \cos (t w) d w \\
& \left.+\int_{2 \pi}^{\frac{8 \pi}{3}} \sqrt{\frac{9 w^{2}}{8 \pi^{2}}-\frac{6 w}{\pi}+8} \cos (t w) d w\right\} .
\end{aligned}
$$

We denote these integrals by $J_{1}, J_{2}, J_{3}$, and $J_{4}$ respectively, and start with $J_{1}$ :

$$
\begin{aligned}
J_{1} & =\frac{3}{\pi^{2} \sqrt{2}} \int_{\frac{2 \pi}{3}}^{\pi} \sqrt{\left(w-\frac{2 \pi}{3}\right)^{2}} \cos (t w) d w=\frac{3}{\pi^{2} \sqrt{2}} \int_{0}^{\frac{\pi}{3}} \gamma \cos t\left(\gamma+\frac{2 \pi}{3}\right) d \gamma \\
& =\frac{3}{\sqrt{2} \pi^{2} t}\left[\frac{\pi}{3} \sin \pi t+\frac{\cos \pi t-\cos (2 \pi t / 3)}{t}\right] .
\end{aligned}
$$

Likewise

$$
\begin{aligned}
J_{4} & =\frac{3}{\pi^{2} 2 \sqrt{2}} \int_{2 \pi}^{\frac{8 \pi}{3}} \sqrt{\left(w-\frac{8 \pi}{3}\right)^{2}} \cos (t w) d w \\
& =\frac{3}{2 \sqrt{2} \pi^{2}} \int_{-\frac{2 \pi}{3}}^{0} \gamma \cos t\left(\gamma+\frac{8 \pi}{3}\right) d \gamma \\
& =\frac{3}{2 \sqrt{2} \pi^{2}}\left[\frac{2 \pi}{3} \frac{\sin 2 \pi t}{t}+\frac{\cos (8 \pi t / 3)-\cos (2 \pi t)}{t^{2}}\right]
\end{aligned}
$$

and

$$
\begin{aligned}
J_{2} & =\frac{3}{\sqrt{2} \pi^{2}} \int_{\pi}^{4 \pi / 3} \sqrt{\frac{2 \pi^{2}}{9}-\left(w-\frac{4 \pi}{3}\right)^{2}} \cos (t w) d w \\
& =\frac{3}{\sqrt{2} \pi^{2}} \int_{-\pi / 3}^{0} \sqrt{\frac{2 \pi^{2}}{9}-\gamma^{2}} \cos t\left(\gamma+\frac{4 \pi}{3}\right) d \gamma \\
& =\frac{\sqrt{2}}{3} \int_{0}^{1 / \sqrt{2}} \sqrt{1-x^{2}} \cos \left(\frac{4 \pi}{3} t-\tau x\right) d x=\frac{\sqrt{2}}{3}\left[\cos \frac{4 \pi t}{3} I_{2}^{\prime}+\sin \frac{4 \pi t}{3} I_{2}^{\prime \prime}\right],
\end{aligned}
$$

where $\tau=\sqrt{2} \pi t / 3$, and $I_{2}^{\prime}, I_{2}^{\prime \prime}$ are given by (7) and (8). Thus

$$
\begin{aligned}
& J_{2}=\frac{1}{3 \sqrt{2}} \cos \left(\frac{4 \pi t}{3}\right) F_{1: 0 ; 0}^{0: 2 ; 0}\left[\begin{array}{cc}
-(-1 / 2),(1) ; \ldots \ldots & \ldots \\
(3 / 2): \ldots & -1,-\pi^{2} t^{2} / 36
\end{array}\right] \\
& +\frac{\pi t}{18 \sqrt{2}} \sin \left(\frac{4 \pi t}{3}\right) F_{1: 0 ; 1}^{0: 2 ; 1}\left[\begin{array}{ccc}
0:(-1 / 2),(1) ; & 1 ; \\
2: \ldots \ldots & (3 / 2) ; & -1,-\pi^{2} t^{2} / 36
\end{array}\right] \text {. }
\end{aligned}
$$


As for $J_{3}$, we have

$$
\begin{aligned}
& J_{3}=\frac{3}{2 \sqrt{2} \pi^{2}} \int_{\frac{4 \pi}{3}}^{2 \pi} \sqrt{\frac{8 \pi^{2}}{9}-\left(w-\frac{4 \pi}{3}\right)^{2}} \cos (t w) d w \\
& =\frac{2 \sqrt{2}}{3} \int_{0}^{1 / \sqrt{2}} \sqrt{1-x^{2}} \cos \left(2 \tau x+\frac{4 \pi t}{3}\right) d x \\
& =\frac{2 \sqrt{2}}{3}\left\{\cos \frac{4 \pi t}{3} I_{2}^{\prime}(2 \tau)-\sin \frac{4 \pi t}{3} I_{2}^{\prime \prime}(2 \tau)\right\} \\
& =\frac{\sqrt{2}}{3} \cos \frac{4 \pi t}{3} F_{1: 0 ; 0}^{0: 2 ; 0}\left[\begin{array}{ccc}
\text { : }(-1 / 2),(1) ; & \ldots & -1,-\pi^{2} t^{2} / 9 \\
(3 / 2): \ldots \ldots & \ldots
\end{array}\right] \\
& -\frac{\sqrt{2} \pi t}{9} \sin \frac{4 \pi t}{3} F_{1: 0 ; 1}^{0: 2 ; 1}\left[\begin{array}{ccc}
0:(-1 / 2),(1) ; & 1 ; & \\
2: \ldots & ; \quad(3 / 2) ; & -1,-\pi^{2} t^{2} / 9
\end{array}\right] .
\end{aligned}
$$

Substituting $J_{i}, i=1,2,3,4$, back into equation (17) yields (13).

The wavelets given in the above theorem are entire functions of exponential type and are given explicitly by Taylor series with known coefficients. This makes it easy to calculate them to any desired degree of accuracy.

\section{REFERENCES}

[1] I. Daubechies, Ten Lectures on Wavelets, SIAM Publications, Soc. Indust. Appl. Math., Philadelphia (1992). MR 93e:42045

[2] A. Erdelyi, W. Magnus, F. Oberhettinger, and F. Tricomi, Higher Transcendental Functions, Vol. 1 McGraw-Hill, New York (1953). MR 15:419i

[3] H. Exton, Multiple Hypergeometric Functions And Applications, John Wiley \& Sons, New York (1976). MR 54:10699

[4] I. Gradshteyn and I. Ryzhik, Tables of Integrals, Series, and Products, Academic Press, New York (1965). MR 33:5952

[5] E. Hernandez and G. Weiss, A First Course on Wavelets, CRC Press, Boca Raton, Florida (1996). MR 97i:42015

[6] H. Srivastava and H. Manocha, A Treatise on Generating functions, John Wiley \& Sons, New York (1984). MR 85m:33016

[7] W.H. Young, On infinite integrals involving a generalization of the sine and cosine functions, Quart. J. Math., Vol. 4 (1912), pp. 161-177.

[8] G. Walter, Translation and dilation invariance in orthogonal wavelets, Appl. Comp. Harmonic Anal., Vol. 1 (1994), pp. 344-349. MR 96b:42040

[9] A. Zayed and G. Walter, Wavelets in Closed Forms, in Wavelet Transforms and Timefrequency Signal Analysis, Appl. Numer. Harmon. Anal., Birkhäuser, Boston, MA, 2001, pp. 121-143. MR 2002c:42061

Department of Mathematical Sciences, DePaul University, Chicago, Illinois 60614

E-mail address: azayed@condor.depaul.edu 